\title{
Using Turn Taking to Mitigate Conflict and Coordination Problems in the Repeated Battle of the Sexes Game *
}

\author{
Sau-Him Paul Lau** and Vai-Lam Mui*** \\ April 2005
}

\begin{abstract}
The Battle of the Sexes game, which captures both conflict and coordination problems, has been applied to a wide range of situations. We show that, by reducing conflict of interests and enhancing coordination, (eventual) turn taking supported by a "turn taking with independent randomizations" strategy allows the players to engage in intertemporal sharing of the gain from cooperation. Using this insight, we decompose the benefit from turn taking into conflict-mitigating and coordination-enhancing components. Our analysis suggests that an equilibrium measure of the "intertemporal degree of conflict" provides an intuitive way to understand the sources of welfare gain from turn taking in the repeated Battle of the Sexes game. We find that when this equilibrium measure is higher, players behave more aggressively and the welfare gain from turn taking is smaller.
\end{abstract}

Key Words: $\quad$ Battle of the Sexes game, turn taking, conflict-mitigating, coordinationenhancing

JEL Classifications: C70, C72

* We are grateful to seminar participants at Monash University for helpful comments, and to Alex Ho and Felix Leung for able research assistance. We thank the Research Grants Council of Hong Kong (project HKU7223/04H) for financial support.

** School of Economics and Finance, University of Hong Kong, Pokfulam, Hong Kong. Email: laushp@hkucc.hku.hk.

*** Department of Economics, Monash University, Clayton, Victoria 3800, Australia. Email: Vai-Lam.Mui@BusEco.Monash.edu.au. 


\section{Introduction}

Economists have used the Battle of the Sexes game, which captures the presence of both conflict and coordination problems in an interesting way, to study a wide range of issues. Selected examples include entry into a market of natural monopoly (Dixit and Shapiro, 1986) and network externality (Besen and Farrell, 1994). A modified version of this game has also been used to study the predictive power of forward induction (Cooper et al., 1993; Muller and Sadanand, 2003).

Table 1 illustrates a popular version of the Battle of the Sexes game. In this version of the game (with the standard assumption of $h>l>0$ ), a couple wants to spend an evening together, but the wife (player 1) prefers to attend a ballet performance, while the husband (player 2) prefers to attend a football match. If they both go to see the ballet performance, the wife gets a payoff of $h$, and the husband gets $l$. If they both go to the football match, the husband gets $h$, and the wife gets $l$. If they choose different activities, each gets a (normalized) payoff of 0 . There are both coordination and conflict elements in this game (see, for example, Friedman, 1994). While both players want to go out together, the conflict element is present because their preferred activities differ, and the coordination element is present because they may end up going to different events if communication between them is limited.

In an early discussion of this game, Luce and Raiffa (1957, p. 94) point out that if the game is played repeatedly, then even when no preplay communication is permitted, players can "signal to each other via their choice patterns on previous plays. Introspectively, we would suspect that, after some preliminary jockeying, the players would settle on a pattern of alternation between [the two efficient outcomes]." In this paper, we study how turn taking can improve the welfare of players in the repeated Battle of the Sexes game by mitigating conflict and coordination problems in the absence of preplay communication. We consider a strategy that we refer to as "turn taking with independent randomizations" (TTIR), in which players randomize independently in the initial periods of the game to determine endogenously when they will embark on the turn-taking path (the coordination problem) as well as who will begin with the good turn (the conflict problem). The TTIR strategy is modified from Crawford and Haller (1990), who consider the use of precedents as focal points in a pure coordination game. By effectively reducing conflict of interest and by eventually solving the coordination problem in the Battle of the Sexes game, turn taking allows players to engage in intertemporal sharing of the gain from cooperation. Using this insight, we decompose the benefit associated with the turn-taking equilibrium into conflict-mitigating and coordination-enhancing components. 
The turn-taking equilibrium we emphasize in this paper is one in which alternation occurs most frequently (i.e., in the turn-taking phase of the equilibrium path, a player takes the good turn in one period, the bad turn in the next, and so on), and we refer to this equilibrium as the "TTIR equilibrium." ${ }^{1} \mathrm{We}$ believe this type of "single-period alternation" is what Luce and Raiffa (1957) refer to in the above quotation and what most people think is meant by turn taking. According to the Folk Theorem of repeated games, there are many possible subgame-perfect equilibria for this game, and the TTIR equilibrium is just one of them. We show that, among different subgame-perfect equilibria with "multiple-period alternation" (i.e., in the turn-taking phase of the equilibrium path, a player takes the good turn in $m$ consecutive periods, the bad turn in the next $m$ consecutive periods, and so on), the TTIR equilibrium (with $m=1$ ) gives the players the highest payoffs. This result provides theoretical justification for the observation that single-period alternation seems to possess some focal-point features. ${ }^{2}$

The key idea unifying our analysis is as follows. For the one-shot version of the Battle of the Sexes game, define $\theta=\frac{h}{l}$ as the degree of conflict of the game. As several authors have argued, in the absence of communication, the logical prediction of this game is the symmetric mixed-strategy equilibrium (see Dixit and Shapiro, 1986, Farrell, 1987; also see Cooper et al., 1989). At the mixed-strategy equilibrium, it can be shown that each of the two players chooses his preferred activity with a probability that depends on $\theta$ only (i.e., it depends only on the ratio of $h$ and $l$ but not on their absolute values). It is clear that $\theta>1$, and thus, each player always chooses his preferred activity with a probability higher than 0.5 at the mixed-strategy equilibrium; see Section 2 and Sub-section 4.1 for details. ${ }^{3}$ In this game, a lower $\theta$ means that the surplus of the game (obtained when the players reach the efficient, instead of the inefficient, outcomes) is more evenly distributed, and thus the degree of conflict is lower. When the degree of conflict $(\theta)$ decreases, the stake in not reaching one's preferred outcome becomes relatively small. As a result, each player chooses his preferred activity with a lower probability, which is closer to 0.5 , and his payoff at the mixed-strategy equilibrium increases. To summarize, the increase in equilibrium payoff for a one-shot Battle of the Sexes game with

\footnotetext{
1 Along the TTIR equilibrium path, there is a turn-taking phase after the players reach one of the efficient outcomes. Prior to that, there is a randomization phase. More detailed analysis is given in Section 3.

2 Whether the single-period turn-taking pattern is observed, of course, can only be confirmed by evidence in the laboratory or in the field. Interestingly, in a study that involves different versions of the game of chicken (which is similar, but not identical, to the Battle of the Sexes game), Bornstein et al. (1997) observe that a significant percentage of subjects use the single-period alternation strategy.

3 The probability of 0.5 is a useful benchmark for comparing players' behavior because it maximizes the likelihood that the players will reach one of the two efficient outcomes in the Battle of the Sexes game.
} 
a lower degree of conflict can be described as conflict mitigating.

The above idea applies to one-shot Battle of the Sexes games with different degrees of conflict. We show that this idea can be generalized to a Battle of the Sexes game with the same parameters $h$ and $l$ (and thus the same $\theta$ ), if repeated play is possible. Specifically, we show that the equilibrium "degree of intertemporal conflict" (in a sense to be made precise later) is lower than the exogenous degree of conflict in the stage game and that the discountedaverage payoff of each player at the TTIR equilibrium is higher than his payoff at the mixed-strategy equilibrium of the stage game. By allowing the players to engage in intertemporal sharing of the gain from cooperation, turn taking reduces the distributional conflict and induces each player to behave less aggressively (by choosing his preferred activity with a probability closer to 0.5 at the randomization phase of the TTIR equilibrium). As a result, players are more likely to reach the efficient turn-taking path in earlier periods of the game, and each player's equilibrium payoff increases. The same intuition also applies when we show that the TTIR equilibrium (with single-period alternation) offers players the highest payoff among all subgame-perfect equilibria with multiple-period alternation. More frequent alternation reduces distributional conflict and induces players to behave less aggressively. Thus, each player's equilibrium payoff is the highest under single-period alternation.

Our analysis suggests that for any $m$-period TTIR equilibrium, there is a naturally defined equilibrium measure of the degree of intertemporal conflict that provides an intuitive way to understand the sources of welfare gain from turn taking in the repeated Battle of the Sexes game. We find that when this equilibrium measure is higher, the players behave more aggressively, and thus the welfare gain from turn taking is smaller. To our knowledge, the question of how the degree of conflict in the stage game or the equilibrium degree of intertemporal conflict in the repeated game affects players' behavior and welfare, has not been addressed in the literature.

This paper is organized as follows. Section 2 introduces the Battle of the Sexes game with symmetric players, and summarizes some well-known results when players interact once. Section 3 obtains the TTIR equilibrium of the repeated Battle of the Sexes game. Section 4 discusses the underlying reasons behind the benefit to players when they use the TTIR strategy, and proposes a way to decompose this benefit into conflict-mitigating and coordination-enhancing components. Section 5 considers the subgame-perfect equilibria with multipleperiod alternation and shows that each player's equilibrium payoff decreases when turn taking occurs less frequently. Section 6 provides concluding remarks. 


\section{One-shot interaction in the Battle of the Sexes game}

We consider the Battle of the Sexes game between two symmetric players (players 1 and 2) with the following payoff structure:

$U_{1}(T, S)=U_{2}(S, T)=h>U_{1}(S, T)=U_{2}(T, S)=l>U_{i}(T, T)=U_{i}(S, S)=0$,

where $T$ (which stands for Tough) and $S$ (which stands for Soft) are the two possible actions for player $i(i=1,2)$, and $U_{i}\left(x_{1}, x_{2}\right)$ is the payoff of player $i$ when player 1 chooses action $x_{1}\left(T\right.$ or $S$ ) and player 2 chooses action $x_{2}$. This game is represented in the left-hand panel of Table 2. To compare this specification of the Battle of the Sexes game with the one illustrated in Table 1, $T$ represents choosing one's preferred activity (ballet for player 1 and football match for player 2), and $S$ represents choosing the other player's preferred activity. ${ }^{4}$

For analysis in later sections, we find it helpful to introduce an alternative specification of the Battle of the Sexes game by defining the following two parameters:

$$
\pi=\frac{h+l}{2}
$$

and

$$
\theta=\frac{h}{l}
$$

Parameter $\pi$, which is positive, can be interpreted as one half of the total surplus if either asymmetric efficient outcome $(T, S)$ or $(S, T)$, rather than $(T, T)$ or $(S, S)$, is reached. ${ }^{5}$ We shall hereafter refer to $\pi$ as the surplus parameter, as this is the maximum attainable (expected) surplus for a player in any symmetric solution of the Battle of the Sexes game. On the other hand, parameter $\theta$ is related to how the total surplus in an efficient outcome is distributed and can be interpreted as the degree of conflict of the game. It is clear that

$$
\theta>1 \text {. }
$$

A higher $\theta$ in this range means that the surplus is more unevenly distributed, and thus the degree of conflict is higher.

\footnotetext{
4 Note that according to this definition, two players choosing the same action (Tough, for example) in the left-hand panel of Table 2 means that they attend different events in Table 1. Cooper et al. (1989) also use the convention of denoting a player's preferred activity (which is different for the two players in the Battle of the Sexes game) as a particular action (action 2 in their Figure 1).

5 One way to see this interpretation of parameter $\pi$ is that if correlated strategy is allowed, then both players may achieve $\pi$ in this game.
} 
It is easy to see from (2) and (3) that

$$
h=\frac{2 \theta \pi}{1+\theta},
$$

and

$$
l=\frac{2 \pi}{1+\theta} .
$$

Using the specification based on the surplus and conflict parameters, the game in (1) can be represented in the right-hand panel of Table 2.

Consider the game described above when the players interact once and choose their actions independently in the absence of preplay communication. It is easy to see that there are two (asymmetric) pure-strategy Nash equilibria: $(T, S)$ and $(S, T)$. There is an obvious source of conflict for the players over these two equilibria, as each player prefers the equilibrium in which he chooses Tough with the other player choosing Soft. There is also a (symmetric) mixedstrategy equilibrium. At this equilibrium, it can be shown that each player chooses Tough with probability $q^{*} \in(0,1)$ to satisfy

$$
q^{*}(0)+\left(1-q^{*}\right)(h)=q^{*}(l)+\left(1-q^{*}\right)(0) .
$$

Equivalently, $q^{*}$ satisfies

$$
\frac{q^{*}}{1-q^{*}}=\frac{h-0}{l-0}=\theta .
$$

As a result, a player's payoff at the mixed-strategy equilibrium is given by

$$
U^{*}=q^{*}\left(1-q^{*}\right)(h+l)=\frac{h l}{h+l}=\frac{2 \theta \pi}{(1+\theta)^{2}} .
$$

One advantage of the specification based on $\pi$ and $\theta$ is that while a player's equilibrium payoff $\left(U^{*}\right)$ depends on both parameters, his behavior $\left(q^{*}\right)$ depends only on the conflict parameter (but not the surplus parameter). This specification is emphasized in the analysis in Section 4. On the other hand, the specification based on $h$ and $l$ is more convenient for some subsequent analyses. We use both specifications in this paper.

\section{The TTIR equilibrium in the repeated Battle of the Sexes game}

We now analyze the Battle of the Sexes game when two symmetric players interact repeatedly. We model the environment in which the players interact repeatedly as an infinite-horizon repeated game with discounting. 
Each of the two players makes a decision, simultaneously and independently, about whether to choose Tough or Soft (or to choose randomly between the two actions) in every period of the game. When making a new decision, say at period $n$, player $i(i=1,2)$ maximizes the discounted average of the stream of his current and future stage-game payoffs (see, for example, Fudenberg and Maskin, 1986), which is given by

$$
(1-\delta) \sum_{r=n}^{\infty} \delta^{r-n} U_{i}\left(x_{1 r}, x_{2 r}\right)
$$

where $\delta \in(0,1)$ is the common discount factor, $x_{i r}\left(x_{i r}=T\right.$ or $\left.S\right)$ is the choice of player $i$ at period $r$, and $U_{i}\left(x_{1 r}, x_{2 r}\right)$ is the stage-game payoff of player $i$ according to (1). To capture the environment in Luce and Raiffa (1957, p. 94), we assume that there is no communication between players (such as nonbinding preplay communication in Farrell, 1987) and that there is no commonly observed variable on which players may condition their strategies and thereby correlate them. Crawford and Haller (1990) also make this assumption in analyzing a pure coordination game.

Analogous to considering the symmetric mixed-strategy equilibrium in the one-shot Battle of the Sexes game (in Section 2), we consider a symmetric subgame-perfect equilibrium of the repeated game in which players use the TTIR strategy. We refer to this equilibrium as the TTIR equilibrium.

The TTIR strategy specifies the following: (a) In the beginning period, the players independently randomize between Tough and Soft. Denote the probability of choosing Tough as $p$, where $p \in(0,1) .{ }^{6}$ (b) As long as the randomization yields the symmetric outcome of either $(T, T)$ or $(S, S)$, the randomization phase will continue. (c) Whenever randomization "succeeds" in getting players to the asymmetric outcome of either $(T, S)$ or $(S, T)$, the game will switch to the turn-taking phase in which each player chooses the action his opponent took in the previous period. If no player defects from this strategy, the turntaking phase will continue. (d) Any defection during the turn-taking phase will trigger a switch to the punishment phase, in which each player uses the equilibrium mixed strategy of the stage game (i.e., each player chooses Tough with probability $q^{*}$ given by $\left.(7)\right)$ in each period. ${ }^{7}$

We now examine whether the above TTIR strategy constitutes a subgame-

\footnotetext{
6 More generally, one can define $p_{i}(i=1,2)$ as the probability that player $i$ chooses Tough in the randomization phase. In the symmetric subgame-perfect equilibrium, the equilibrium values of $p_{1}$ and $p_{2}$ are equal. To avoid heavy use of notations, we specify from the beginning that both players use the same strategy. There is no loss of generality since we consider only symmetric equilibrium.

7 In Section 6, we consider other punishment strategies. We show that our results continue to hold under these strategies.
} 
perfect equilibrium. Define $W^{H}$ as the player's discounted-average payoff at a period in which he chooses Tough and the other player chooses Soft, with the expectation that players will choose the equilibrium TTIR strategy forever. Similarly, define $W^{L}$ as the player's discounted-average payoff at a period in which he chooses Soft and the other player chooses Tough, with the expectation that players will choose the equilibrium TTIR strategy forever. Finally, define $W^{*}$ as the value of the game, which is a player's discounted-average payoff at the initial period or any period in the randomization phase (such that both players' actions were the same in the previous period), with the expectation that players will choose the equilibrium TTIR strategy forever.

It is straightforward to show that for the Battle of the Sexes game the value functions at the turn-taking phase are given by

$$
W^{H}=(1-\delta) h+\delta W^{L}=\frac{h+\delta l}{1+\delta}=\frac{2(\delta+\theta) \pi}{(1+\delta)(1+\theta)}
$$

and

$$
W^{L}=(1-\delta) l+\delta W^{H}=\frac{l+\delta h}{1+\delta}=\frac{2(1+\delta \theta) \pi}{(1+\delta)(1+\theta)}
$$

To ensure that (9) and (10) are well defined, we need to verify that players will not deviate from the equilibrium strategy. Because of the stationary structure of the infinite-horizon repeated game, it is necessary to check only two nodeviation conditions at the turn-taking phase, one at the player's good turn when he is supposed to play Tough, and the other at the player's bad turn when he is supposed to play Soft. The no-deviation condition at a player's good turn (when the actions of the player and his opponent were Tough and Soft, respectively, in the previous period) is satisfied, because ${ }^{8}$

$$
W^{H}-\delta U^{*}=(1-\delta)(h-0)+\delta\left(W^{L}-U^{*}\right)>0 .
$$

Similarly, the no-deviation condition at a player's bad turn is satisfied, because

$$
W^{L}-\delta U^{*}=(1-\delta)(l-0)+\delta\left(W^{H}-U^{*}\right)>0 .
$$

Now, examine the beginning of the game (or any period in the randomization phase). If both players use the equilibrium strategy in every period, it is easy to see that the game will remain in the randomization phase in the next period if and only if both players happen to choose the same action in the current period. As a result, the payoff matrix of the repeated game (when viewed at the beginning period) is given by Table 3 .

\footnotetext{
8 From (8), it is easy to show that $U^{*}<l<h$. Since $W^{L}$ is a weighted average of $h$ and $l$, it is obvious that $W^{L}>U^{*}$. Thus, (11) holds. Similarly, (12) holds because $W^{H}$ is a weighted average of $h$ and $l$.
} 
For subsequent analysis, it is helpful to define the following function:

$$
W(p)=\frac{p(1-p)\left(W^{H}+W^{L}\right)}{1-\delta\left[p^{2}+(1-p)^{2}\right]}=\frac{2 p(1-p) \pi}{1-\delta\left[p^{2}+(1-p)^{2}\right]}
$$

If both players choose Tough with probability $p$ in the randomization phase, then this phase will continue with probability $p^{2}+(1-p)^{2}$ in the next period. Therefore, the function $W(p)$ represents a player's discounted-average payoff at the randomization phase when both players choose Tough with probability $p$.

Denote the equilibrium probability of choosing Tough in the randomization phase as $p^{*}$. We hereafter refer to $p^{*}$ as the equilibrium randomization probability. When both players use the equilibrium mixed strategy in the randomization phase, it can be deduced from Table 3 that the value of the game $W^{*}$ and the equilibrium randomization probability $p^{*}$ are jointly determined by

$$
W^{*}=p^{*}\left(\delta W^{*}\right)+\left(1-p^{*}\right)\left(W^{H}\right)=p^{*}\left(W^{L}\right)+\left(1-p^{*}\right)\left(\delta W^{*}\right) .
$$

Note that $W^{*}$, given by (14) or (A2) in the Appendix, is related to the function $W(p)$ in $(13)$ according to

$$
W^{*}=W\left(p^{*}\right)
$$

In the TTIR equilibrium, a player chooses $p^{*}$ to make the other player indifferent between playing Tough and Soft. In the Appendix, it is shown that (14) leads to ${ }^{9}$

$$
p^{*}=\frac{W^{H}-\delta W^{*}}{\left(W^{H}-\delta W^{*}\right)+\left(W^{L}-\delta W^{*}\right)}=\frac{\frac{2(\delta+\theta) \pi}{(1+\delta)(1+\theta)}-\delta\left\{\frac{2 p^{*}\left(1-p^{*}\right) \pi}{1-\delta\left[\left(p^{*}\right)^{2}+\left(1-p^{*}\right)^{2}\right]}\right\}}{2 \pi-2 \delta\left\{\frac{2 p^{*}\left(1-p^{*}\right) \pi}{1-\delta\left[\left(p^{*}\right)^{2}+\left(1-p^{*}\right)^{2}\right]}\right\}} .
$$

Equation (15) can be interpreted as follows. A simple manipulation of the second equality of (14) shows that the equilibrium randomization probability $p^{*}$ in the current period is given by the middle term of (15), which involves $W^{*}$, since the game may remain in the randomization phase in the next period. According to (A2) in the Appendix, a player's continuation payoff in the randomization phase, which is also equal to $W^{*}$ because of the stationary structure of the game, depends on (future) $p^{*}$. Thus, one can think of $p^{*}$ on the left-hand side of (15) as the probability that both players choose Tough in the current period (at the randomization phase), and $p^{*}$ on the right-hand side as the probability that both players choose Tough in the future if the game

$\overline{9}$ While we can simplify the right-hand term of $(15)$, we choose to use this form so
that the relationship of this term with $W^{H}, W^{L}$, and $W^{*}$ can be seen more clearly. 
remains in the randomization phase. The equilibrium condition (15) - which says that $p^{*}$ has to satisfy a fixed point requirement - can be regarded as a consistency condition between current and future randomization probabilities of this infinitely repeated game with discounting.

In summary, for the repeated Battle of the Sexes game, a TTIR equilibrium exists if there exists a $p^{*} \in(0,1)$ that satisfies the equilibrium randomization condition (15). Moreover, the TTIR equilibrium is unique if there exists only one $p^{*} \in(0,1)$ that satisfies this condition.

In the Appendix, we show that the TTIR equilibrium exists and is unique for the repeated Battle of the Sexes game. The result is summarized in the following Proposition.

Proposition 1 For the infinitely-repeated Battle of the Sexes game with discounting, there exists a value of $p^{*} \in(0,1)$ such that the strategy profile in which both players adopt TTIR constitutes a subgame-perfect equilibrium for all discount factors $\delta \in(0,1)$. Moreover, this equilibrium value is unique. The unique equilibrium randomization probability satisfies

$$
0.5<p^{*}<1
$$

and is given by

$$
p^{*}=\frac{\sqrt{(1+2 \delta+\theta)^{2}+4 \delta(\theta-1)(\delta+\theta)}-(1+2 \delta+\theta)}{2 \delta(\theta-1)} .
$$

\section{Understanding the welfare gain of turn taking in the Battle of the Sexes game}

In the previous section we discuss how (eventual) turn taking can be supported by TTIR as a subgame-perfect equilibrium for the repeated Battle of the Sexes game. In this section we discuss complementary questions. For this game, what is the benefit of repeated interaction when players use the TTIR strategy? What are the underlying reasons for this welfare gain?

It turns out that an analysis of the one-shot Battle of the Sexes game with different degrees of conflict provides insights into the benefit of turn taking. Thus, we first focus on the one-shot Battle of the Sexes game in Sub-section 4.1. We then return to the repeated game and examine players' equilibrium behavior and welfare, respectively, in Sub-sections 4.2 and 4.3. The magnitude of the welfare gain will be discussed in Sub-section 4.4. 


\subsection{One-shot Battle of the Sexes games with different degrees of conflict}

In this sub-section, we consider one-shot Battle of the Sexes games with the same surplus parameter but different conflict parameters. We examine how a change in the conflict parameter affects players' behavior and welfare. To make his opponent willing to randomize in the one-shot Battle of the Sexes game, a player chooses Tough with probability $q^{*}$ to satisfy $(7)$. It is clear from (7) that $q^{*}$ depends only on the degree of conflict $(\theta)$ of the game.

First, we observe from (4) and (7) that

$$
0.5<q^{*}<1 \text {. }
$$

Since $\theta>1$, if a player chooses, say, $q^{*}=0.5$, then the other player will strictly prefer playing Tough and will not be willing to randomize. In equilibrium, each player chooses $q^{*}>0.5$ to ensure that the other player is willing to randomize between Tough and Soft.

Second, it can be shown from (7) that

$$
\frac{d q^{*}}{d \theta}>0
$$

When the degree of conflict increases, the stake in not reaching one's preferred outcome is relatively high. As a result, each player has to choose a higher value of $q^{*}$ to ensure that the other player is willing to randomize.

To see how players' behavior affects their payoffs in the one-shot Battle of the Sexes game, we define

$$
U(q)=q(1-q)(h+l)=2 q(1-q) \pi .
$$

When both players choose Tough with probability $q$, each player's payoff is given by $U(q)$. It is easy to see from $(20)$ that $U(q)$ is a quadratic function of $q$ with the maximum at $q=0.5$ and decreasing in either direction away from 0.5. That is, the slope function $U^{\prime}(q)$ is positive in $q \in(0,0.5)$ and negative in $q \in(0.5,1)$.

Third, $U^{*}$ in (8) is related to $U(q)$ according to

$$
U^{*}=U\left(q^{*}\right) .
$$

Combining the above results, we obtain

$$
\frac{d U^{*}}{d \theta}=U^{\prime}\left(q^{*}\right) \frac{d q^{*}}{d \theta}<0 .
$$


The intuition of (21) is as follows. When the degree of conflict $(\theta)$ of the Battle of the Sexes game decreases, $q^{*}$ decreases in the range $(0.5,1)$ and becomes closer to (but is still higher than) 0.5 , leading to a higher probability, $2 q^{*}\left(1-q^{*}\right)$, of getting to the efficient outcome of either $(T, S)$ or $(S, T)$. This change in players' behavior $\left(q^{*}\right)$ leads to an increase in each player's payoff $\left(U^{*}\right)$, because $q^{*}>0.5$ and $U^{\prime}\left(q^{*}\right)$ is negative.

We represent these results in Figure 1. The points are drawn for a given value of $\pi(\pi=100)$. At a particular $\theta$ (say, $\theta=4)$ of the Battle of the Sexes game, point $A$ represents $(h, l)=\left(\frac{2 \theta \pi}{1+\theta}, \frac{2 \pi}{1+\theta}\right)=(160,40)$ and point $B$ represents $(l, h)=\left(\frac{2 \pi}{1+\theta}, \frac{2 \theta \pi}{1+\theta}\right)=(40,160)$. They are the pure-strategy equilibria of the one-shot Battle of the Sexes game. The various outcomes of this game are represented by point $O$ (the origin), point $A$, and point $B$. Moreover, players' payoffs at the mixed-strategy equilibrium, $\left(U^{*}, U^{*}\right)=(32,32)$, are represented by point $C$ on the 45 -degree line.

Consider another game with the same $\pi$, but $\theta^{\prime}=1.5<\theta$ (i.e., a lower degree of conflict). The various payoffs of this game are represented by points $O, A^{\prime}$, and $B^{\prime}$, where $A^{\prime}$ represents $(120,80)$ and $B^{\prime}$ represents $(80,120)$. It is easy to see that $A^{\prime}$ and $B^{\prime}$ lie on line segment $A B$ (as $\pi$ is unchanged), but the length of $A^{\prime} B^{\prime}$ is shorter than that of $A B$. For this game, players' payoffs at the mixed-strategy equilibrium are given by $(48,48)$ and represented by point $C^{\prime}$.

Two observations can be obtained from Figure 1. First, when $\theta$ decreases, lines $O A$ and $O B$ are rotated toward the 45-degree line (to $O A^{\prime}$ and $O B^{\prime}$, respectively), because of (3). Second, when $\theta$ decreases, the player's payoff at the mixed-strategy equilibrium increases from $O C$ to $O C^{\prime}$, according to (21).

\subsection{Randomization probability at the turn-taking equilibrium}

In the one-shot Battle of the Sexes game, we see that a change in the degree of conflict $(\theta)$ affects players' behavior $\left(q^{*}\right)$ and thus their payoff $\left(U^{*}\right)$. We now examine how this insight can be extended to the TTIR equilibrium of the repeated game. To examine the effect of repeated interaction in the Battle of the Sexes game, we consider a change in the discount factor (from 0 to a positive number less than 1) but no change in the conflict parameter.

In the following Proposition, we compare the probability of choosing Tough $\left(q^{*}\right)$ at the mixed-strategy equilibrium of the one-shot Battle of the Sexes game with the probability of choosing Tough $\left(p^{*}\right)$ at the TTIR equilibrium of the repeated game. As mentioned before, a player chooses $q^{*}>0.5$ according to $(7)$ to make the other player willing to randomize in the one-shot game. 
Similarly, to make the other player willing to randomize in the repeated game, a player chooses $p^{*}>0.5$ to ensure that the second equality of (14) holds. ${ }^{10}$

Proposition 2 In the TTIR equilibrium of the repeated Battle of the Sexes game, the probability of choosing Tough $\left(p^{*}\right)$ is always lower than the probability of choosing Tough $\left(q^{*}\right)$ at the mixed-strategy equilibrium of the one-shot game. That is,

$$
0.5<p^{*}<q^{*}<1
$$

The proof of Proposition 2, which is guided by a comparison of the distributional conflict between players at the one-shot game versus that at the repeated game, is given in the Appendix. Note that if players of the one-shot Battle of the Sexes game reach an asymmetric efficient outcome, one player gets $h$, and the other gets $l$. If they fail to achieve an efficient outcome, each player gets zero. Therefore, the ratio of one player's larger gain from cooperation $(h-0)$ to the other player's smaller gain from cooperation $(l-0)$ is given by the degree of conflict in the stage game; that is, $\theta=\frac{h-0}{l-0}$. According to (7), the odds in favor of Tough (i.e., the probability ratio $\frac{q^{*}}{1-q^{*}}$ ) at the mixed-strategy equilibrium of the one-shot Battle of the Sexes game is given by $\theta \cdot{ }^{11}$ On the other hand, the odds in favor of Tough $\left(\frac{p^{*}}{1-p^{*}}\right)$ at the randomization phase of the TTIR equilibrium are given by

$$
\frac{p^{*}}{1-p^{*}}=\frac{W^{H}-\delta W^{*}}{W^{L}-\delta W^{*}} \equiv \theta^{R}
$$

which is obtained from the second equality of (14). The middle term of (23) has an interpretation similar to (7) of the one-shot game. When players use the TTIR strategy in the repeated game, each player gets an expected payoff of $\delta W^{*}$ in the continuation subgame if they fail to reach an efficient outcome in a particular period. Therefore, $W^{H}-\delta W^{*}$ is one player's larger gain from intertemporal cooperation (if they reach the turn-taking path) and $W^{L}-\delta W^{*}$ is the other player's smaller gain. The middle term of (23), which is the ratio of these two gains, can be interpreted as the degree of intertemporal conflict at the TTIR equilibrium of the repeated Battle of the Sexes game. We denote this term by $\theta^{R}$, where $R$ refers to the repeated game. Because the equilibrium

$\overline{10}$ The intuition behind $p^{*}>0.5$ in (16) is similar to that behind $q^{*}>0.5$ in (18) of the one-shot game. In the randomization phase, a player chooses $p^{*}$ to make the other player indifferent between playing Tough and Soft. Since $W^{H}>W^{L}$ according to (4), (9), and (10), if a player chooses, say, $p^{*}=0.5$, then the other player will strictly prefer playing Tough and will not be willing to randomize. In equilibrium, each player chooses $p^{*}>0.5$ to ensure that the other player is indifferent between choosing Tough or Soft.

11 The odds of a particular event represent the probability of that event occurring, as opposed to something else occurring. See, for example, Dixit and Skeath (2004, p. 186, footnote 1) for more discussion. 
values of $p^{*}$ and $W^{*}$ are determined simultaneously as functions of the primitives of the repeated game, it is clear that $\theta^{R}$ is determined endogenously as a function of these primitives. Since $p^{*}$ only depends on $\theta$ and $\delta$ according to (17) and $\theta^{R}=\frac{p^{*}}{1-p^{*}}$, the equilibrium degree of intertemporal conflict depends only on the exogenous degree of conflict in the stage game and on players' impatience.

The intuition of Proposition 2 is that the equilibrium degree of intertemporal conflict of the repeated Battle of the Sexes game is less than the original degree of conflict of the one-shot game; that is, $\theta^{R}<\theta$. In the turn-taking equilibrium, even though a player is currently taking the bad turn while the other player takes the good one, the distributional conflict is less severe, since the first player knows that with repeated play he will take the good turn in the next period (and also periodically in other future periods). As a result of the less severe distributional conflict, $p^{*}$ is closer to 0.5 when compared to $q^{*}$.

\subsection{Conflict-mitigating and coordination-enhancing benefits of turn taking}

According to Proposition 2, the probability of choosing Tough at the TTIR equilibrium of the repeated Battle of the Sexes game is lower than the probability of choosing Tough at the mixed-strategy equilibrium of the one-shot game, because the (equilibrium) degree of intertemporal conflict is reduced by turn taking. In this sub-section, we examine how this change in behavior affects players' payoffs, and we provide a way to understand the welfare gain of turn taking in the Battle of the Sexes game.

We now compare each player's discounted-average payoff at the TTIR equilibrium of the repeated game $\left(W^{*}\right)$ with his payoff at the mixed-strategy equilibrium of the one-shot game $\left(U^{*}\right)$. Using (13) and (20), the payoff differential can be decomposed as the sum of the following two terms:

$$
W^{*}-U^{*}=W\left(p^{*}\right)-U\left(q^{*}\right)=\left[U\left(p^{*}\right)-U\left(q^{*}\right)\right]+\left[W\left(p^{*}\right)-U\left(p^{*}\right)\right] .
$$

The first component of the payoff differential can be understood as follows. If players do not engage in turn taking and just randomize with probability $q^{*}$ every period, each will get a discounted-average payoff equal to the mixedstrategy equilibrium payoff $U^{*}=2 q^{*}\left(1-q^{*}\right) \pi$ in the stage game, which can be denoted as $U\left(q^{*}\right)$. On the other hand, if they randomize with probability $p^{*}$ every period, each will get a discounted-average payoff $U\left(p^{*}\right)=2 p^{*}\left(1-p^{*}\right) \pi$.

Since $0.5<p^{*}<q^{*}<1$ and $U^{\prime}(q)<0$ for $0.5<q<1$, we conclude that

$$
U\left(p^{*}\right)-U\left(q^{*}\right)=2\left[p^{*}\left(1-p^{*}\right)-q^{*}\left(1-q^{*}\right)\right] \pi>0 .
$$


The intuition of (25) is as follows. Recall that $q^{*}$ is determined by the degree of conflict $(\theta)$ of the stage game according to $(7)$, while $p^{*}$ is determined by the equilibrium degree of intertemporal conflict $\left(\theta^{R}\right)$ in the repeated game according to (23). We show in Proposition 2 that $\theta^{R}<\theta$, and thus, $0.5<$ $p^{*}<q^{*}<1$. As a result, each player's payoff when both randomize with $p^{*}$ each period is higher than the payoff each gets when both randomize with $q^{*}$ each period. Because this increase in payoff is similar to that associated with a decrease in the degree of conflict in the one-shot game (see Figure 1), we call this component of payoff increase associated with turn taking the conflict-mitigating benefit.

Of course, in the TTIR equilibrium, players do not randomize with probability $p^{*}$ every period. According to the TTIR strategy, players will switch from the randomization phase to the turn-taking phase when randomization leads to the asymmetric outcome of either $(T, S)$ or $(S, T)$ in a particular period. Besides the conflict-mitigating benefit in (25), turn taking also delivers another benefit to players of the repeated Battle of the Sexes game. This benefit is given by the second term in the right-hand side of (24). It is easy to see that

$$
W\left(p^{*}\right)=\frac{2 p^{*}\left(1-p^{*}\right) \pi}{1-\delta\left[\left(p^{*}\right)^{2}+\left(1-p^{*}\right)^{2}\right]}>2 p^{*}\left(1-p^{*}\right) \pi=U\left(p^{*}\right)
$$

In the repeated Battle of the Sexes game, if each of the two players uses the equilibrium TTIR strategy, then they will reach the efficient outcome of either $(T, S)$ or $(S, T)$ after some initial periods of "trial and error." Not surprisingly, a player's payoff at the TTIR equilibrium, $W^{*}=W\left(p^{*}\right)$, is higher than $U\left(p^{*}\right)$, his payoff when both players do not engage in turn taking and simply randomize with probability $p^{*}$ each period. Since $W\left(p^{*}\right)-U\left(p^{*}\right)$ is related to the fact that the TTIR strategy allows players to reach the efficient asymmetric outcomes even without preplay communication, we call it the coordination-enhancing benefit.

We summarize the benefits of turn taking in the repeated Battle of the Sexes game in the following Proposition.

Proposition 3. Compared with the payoff at the mixed-strategy equilibrium in the one-shot Battle of the Sexes game, a player's discounted-average payoff at the TTIR equilibrium of the repeated game (with the same surplus and conflict parameters) is higher. Moreover, this payoff differential can be decomposed as the sum of the conflict-mitigating benefit and the coordination-enhancing benefit, according to (24).

The benefit of the TTIR strategy for the repeated Battle of the Sexes game can be illustrated graphically as follows. Figure 2 is drawn for a fixed value of $\pi=100$. For easy comparison with Figure 1 , the payoff points $A, B$ (and 
the origin) correspond to the game with $\theta=4$. As before, players' payoffs at the mixed-strategy equilibrium of the one-shot game are given by point $C$. When repetition becomes relevant and $\delta$ increases from zero (to, say, 0.78125), players' equilibrium payoffs - when evaluated at any period during the turntaking phase - are represented by points $A_{\delta}$ and $B_{\delta}$. Note that, similar to Figure 1, lines $O A$ and $O B$ are rotated toward the 45-degree line to $O A_{\delta}$ and $O B_{\delta}$. Moreover, players' equilibrium discounted-average payoffs (when evaluated in the beginning of the repeated game), $\left(W^{*}, W^{*}\right)=(80.84,80.84)$, are represented by point $C_{\delta}$. According to Proposition $3, W^{*}>U^{*}$, and the payoff increase $\left(W^{*}-U^{*}\right)$ associated with turn taking is represented by the distance $C C_{\delta}$. Furthermore, this payoff increase can be decomposed as the sum of $C C^{\prime}$ (the conflict-mitigating benefit) and $C^{\prime} C_{\delta}$ (the coordination-mitigating benefit). ${ }^{12}$

\subsection{The magnitude of the welfare gain}

We have obtained qualitative results pertaining to the welfare gain of turn taking in Proposition 3, and we now examine the magnitude of the welfare gain. For selected parameter values of $\theta$ and $\delta$ (at a given value of $\pi=100$ ), the equilibrium values of $p^{*}$ are given in Table 4 , the equilibrium intertemporal degrees of conflict $\left(\theta^{R}\right)$ are given in Table 5 , and the corresponding values of $W^{*}$ are given in Table 6 . Note that by fixing $\pi=100$, the entry in Table 6 can be interpreted as the percentage of maximum surplus $(\pi)$ obtained.

One way to observe the benefit of repetition with turn taking supported by the TTIR strategy in Table 6 is to fix the value of $\theta$ (say, $\theta=4$ and thus $h=4 l$ ) and compare a player's payoff in the one-shot game (the entry in the last row) with that in another row with $\delta>0$ (say, $\delta=0.9$ ). It is easy to conclude that players' welfare is higher in the repeated game.

Quantitatively, the effect of repetition on $W^{*}$ can be quite substantial. Consider, again, $\theta=4$. When players interact once $(\delta=0)$, only $32 \%$ of a player's maximum surplus $(\pi)$ is obtained at the mixed-strategy equilibrium. On the other hand, by allowing repetition $(\delta>0)$ only, the increase in players' welfare can be huge, especially when they are relatively patient. As an example, when $\delta$ is 0.78125 , the equilibrium degree of intertemporal conflict is $\theta^{R}=\frac{p^{*}}{1-p^{*}}=1.5$, which is smaller than the exogenous degree of conflict of the stage game $(\theta=4)$. In this case, a player's discounted-average payoff in the repeated game is 80.84, which is more than double his payoff (32.00) in the

\footnotetext{
$\overline{12}$ Note that by choosing $\delta=\frac{25}{32}=0.78125$, both $C^{\prime}$ in Figure 2 (corresponding to $p^{*}=0.6$ in the repeated game when $\theta=4$ ) and $C^{\prime}$ in Figure 1 (corresponding to $q^{*}=0.6$ in the one-shot game when $\left.\theta=1.5\right)$ represent the point $(48,48)$.
} 
one-shot game. The increase in players' welfare is even more dramatic when $\theta$ is large, as can be observed in the last column of Table 6 .

In Table 7, the welfare gain of turn taking is decomposed into conflict-mitigating and coordination-enhancing components. We observe from Table 7 that turn taking can lead to huge coordination-enhancing benefits, especially if the discount factor is high. Moreover, the ratio of coordination-enhancing benefit to conflict-mitigating benefit varies with $\delta$ and $\theta$, with a lower proportion of coordination-enhancing benefit occurring when the degree of conflict is higher and players are relatively impatient.

\section{Multiple-period alternation}

In the TTIR equilibrium studied in earlier sections, alternation occurs every period (in the turn-taking phase). We believe this pattern is what Luce and Raiffa (1957) and most people have in mind about turn taking in the Battle of the Sexes game. From the Folk Theorem of repeated games with discounting (see, for example, Fudenberg and Maskin, 1986, 1991; Abreu, 1988), we know that the TTIR equilibrium is just one of many possible subgame-perfect equilibria for this game. In this section, we provide some justification for the TTIR equilibrium by showing that players' welfare at the TTIR equilibrium (with single-period alternation) is the highest among all symmetric subgame-perfect equilibria with multiple-period alternation.

As in Section 3, there is no preplay communication between players, and we consider a strategy that is similar to the TTIR strategy. In particular, there is a randomization phase in the beginning. The only difference is in step (c) of the TTIR strategy. For the multiple-period turn taking with independent randomizations (hereafter multiple-period TTIR) strategy considered in this section, we specify that, as long as the randomization yields the asymmetric efficient outcome of either $(T, S)$ or $(S, T)$, the player who takes the good turn will continue to take the good turn for another $m-1$ periods, where $m$ is an integer larger than or equal to 1. Afterwards, the other player takes the good turn for $m$ periods. And so on.

Since the analysis in this section is quite similar to that in Section 3, we keep the presentation brief. Define $W_{m}^{H}$ as the player's discounted-average payoff at the first period in which he chooses Tough and the other player chooses Soft, with the expectation that players will choose the equilibrium multipleperiod TTIR strategy in all future periods. Similarly, define $W_{m}^{L}$ as the player's discounted-average payoff at the first period in which he chooses Soft and the other player chooses Tough, with the expectation that players will choose the 
equilibrium strategy forever. ${ }^{13}$ Finally, define $W_{m}^{*}$ as a player's discountedaverage payoff at any period in the randomization phase, with the expectation that players will choose the equilibrium strategy forever.

With the same punishment strategy as in Section 3, it can be shown that the players will not deviate from the equilibrium strategy in the turn-taking phase. Moreover, it is easy to see that

$$
W_{m}^{H}=\frac{h+\delta^{m} l}{1+\delta^{m}},
$$

and

$$
W_{m}^{L}=\frac{l+\delta^{m} h}{1+\delta^{m}}
$$

Denote the equilibrium probability of choosing Tough in the randomization phase as $p_{m}^{*}$. It can be shown that $W_{m}^{*}$ and $p_{m}^{*}$ are jointly determined by

$$
W_{m}^{*}=p_{m}^{*}\left(\delta W_{m}^{*}\right)+\left(1-p_{m}^{*}\right)\left(W_{m}^{H}\right)=p_{m}^{*}\left(W_{m}^{L}\right)+\left(1-p_{m}^{*}\right)\left(\delta W_{m}^{*}\right) .
$$

In the Appendix, we prove the following Proposition.

Proposition 4. (a) For the repeated Battle of the Sexes game, there exists a value of $p_{m}^{*} \in(0,1)$ such that the strategy profile in which both players adopt multiple-period TTIR constitutes a subgame-perfect equilibrium for all discount factors $\delta \in(0,1)$. Moreover, this equilibrium value is unique, and it satisfies

$$
0.5<p_{m}^{*}<1 .
$$

(b) $p_{m}^{*}$ is increasing in $m$. (c) $W_{m}^{*}$ is decreasing in $m$.

The intuition of Proposition 4 is as follows. We rearrange (29) to obtain

$$
\frac{p_{m}^{*}}{1-p_{m}^{*}}=\frac{W_{m}^{H}-\delta W_{m}^{*}}{W_{m}^{L}-\delta W_{m}^{*}} \equiv \theta_{m}^{R} .
$$

The odds in favor of Tough $\left(\frac{p_{m}^{*}}{1-p_{m}^{*}}\right)$ at the randomization phase are given by the middle term in (31). Analogous to (7) and (23), we can interpret the middle term of (31) - the ratio of the two players' gains from intertemporal cooperation - as the equilibrium degree of intertemporal conflict $\left(\theta_{m}^{R}\right)$ of the repeated Battle of the Sexes game when the players use the multiple-period TTIR strategy. Note that analogous to the fact that $\theta^{R}$ in (23) depends only on $\theta$ and $\delta$, it can be shown that $\theta_{m}^{R}$ depends only on $\theta, \delta$, and $m$. Comparing (23) with (31), it is obvious that $\theta_{m}^{R}=\theta^{R}$ when $m=1$.

\footnotetext{
$\overline{13}$ Note that the other $2 m-2$ value functions at the turn-taking phase can be similarly defined, but they are not essential for the subsequent analysis.
} 
The key point of Proposition 4 is that the equilibrium degree of intertemporal conflict of the Battle of the Sexes game is different for turn taking of different length of alternation. Proposition 4(b) establishes that the equilibrium degree of intertemporal conflict is increasing in the length of alternation. When $m$ increases, turn taking becomes less frequent, and thus the importance of being the first one to take the good turn increases. Therefore, an increase in the length of alternation induces each player to behave more aggressively in the randomization phase, and thus the equilibrium value of $p_{m}^{*}$ increases and is further from 0.5. Furthermore, much as we do in earlier sections, we show in Proposition 4(c) that players' more aggressive behavior increases the length of time it is expected to take to reach the turn-taking path, which reduces the welfare of both players. As a result, $W_{m}^{*}$ is decreasing in $m$.

To summarize, a TTIR strategy with a different length of alternation can be viewed as a different mechanism for the intertemporal sharing of gain from cooperation in the repeated Battle of the Sexes game. The intuition of Proposition 4 is that the equilibrium degree of intertemporal conflict $\left(\theta_{m}^{R}\right)$ of such a mechanism provides a parsimonious and intuitive way for understanding its efficacy in mitigating coordination and conflict problems. When an intertemporal gain-sharing mechanism is associated with a higher degree of intertemporal conflict, players behave more aggressively as a result of their efforts to capture a larger share of the intertemporal welfare gain. Consequently, this mechanism gives players a lower payoff compared to another mechanism associated with a lower degree of intertemporal conflict.

This insight is related to, and extends, an important finding in Bhaskar (2000). In the context of the repeated Battle of the Sexes game and the repeated Hawk-and-Dove game, he compares the efficiency properties of two "conventions," where a convention is defined as "a rule which achieves asymmetric coordination by conditioning upon history" (Bhaskar, 2000, p. 250). In that paper, the players also use independent randomizations to resolve the coordination problem. A "bourgeois" convention specifies that after an asymmetric outcome is reached as a result of randomization in some initial period, players will choose this particular outcome in all future periods. A convention is "egalitarian" if players' payoffs are equalized as much as possible. ${ }^{14}$ The key point of Bhaskar's analysis is that different conventions give rise to different

\footnotetext{
${ }^{14}$ The egalitarian convention works as follows (Bhaskar, 2000, pp. 256-257). Suppose the realized outcome in some initial period is $(T, S)$, and player 1's currentperiod payoff exceeds player 2's. To implement the egalitarian convention, the players choose $(S, T)$ in succeeding periods until the discounted-average payoff of player 2 exceeds that of player 1 . At this point the players switch to playing $(T, S)$ until player 1's discounted-average payoff exceeds player 2's, and so on. This method, which is an application of the results in Sorin (1986) and Fudenberg and Maskin (1991), is based on the idea of keeping track of players' discounted-average payoffs at each period.
} 
incentives for players' behavior in the randomization phase. Under the bourgeois convention, each player has a high stake in ensuring that coordination will be achieved in his preferred asymmetric outcome, and this causes players to place more weight on playing Tough in the randomization phase than they do under the egalitarian convention. As a result, coordination is less likely to be achieved in earlier periods under the bourgeois convention, and this convention is relatively less efficient.

The results in Bhaskar (2000) are closely related to the insight of our Proposition 4. Bhaskar's finding can be framed as saying that because the bourgeois convention involves a higher degree of intertemporal conflict than the egalitarian convention, it causes players to behave more aggressively and hence leads to lower welfare than the egalitarian convention. ${ }^{15}$ Despite this similarity, there are major differences between this paper and Bhaskar (2000). Motivated by the observation that turn taking is often used as a mechanism of intertemporal sharing of gains from cooperation, we consider the TTIR strategy that specifies periodic alternation between players once they embark on the turntaking path. The egalitarian convention considered by Bhaskar, on the other hand, involves the use of more complex time-varying strategies which equalizes players' payoffs (see footnote 14). Moreover, this paper demonstrates the importance of the degree of conflict in the stage game and the equilibrium degree of intertemporal conflict in the repeated game in understanding the efficacy of different mechanisms in mitigating conflict and coordination problems. This insight, which to our knowledge has not been emphasized in the literature, provides a unified way of understanding the superiority of the egalitarian convention relative to the bourgeois convention in Bhaskar (2000) and the benefit from turn taking analyzed in this paper.

\section{Conclusion}

We conclude by discussing three issues: the possibility of alternative punishment, the efficiency loss in the TTIR equilibrium, and directions for future research.

In our specification of the TTIR strategy, we assume that defection during the turn-taking phase will trigger the play of the static mixed-strategy equilibrium forever. As we now demonstrate, our results regarding the equilibrium randomization probability $\left(p^{*}\right)$ and the player's equilibrium payoff $\left(W^{*}\right)$ continue to hold whenever an alternative punishment strategy prevents players from deviating from equilibrium behavior. For example, consider a new TTIR strategy

\footnotetext{
$\overline{15}$ Note that when $m \rightarrow \infty$, the TTIR strategy with $m$-period alternation studied in this section becomes the bourgeois convention in Bhaskar (2000).
} 
with an alternative punishment strategy that replaces step (d) in Section 3 with the following: Any defection during the turn-taking phase will trigger a switch back to the randomization phase, and this randomization phase will continue until randomization succeeds in getting players to the asymmetric outcome of either $(T, S)$ or $(S, T)$ again. ${ }^{16}$ Provided that the no-deviation conditions during the turn-taking phase are satisfied, it is easy to see that the analysis in the randomization phase is the same as in Section 3. In particular, $p^{*}$ and $W^{*}$, which are related by (14), are the same as before. From (A4) and (A5) of the Appendix, we conclude that the two no-deviation conditions of the TTIR strategy supported by this alternative punishment strategy are indeed satisfied. That is,

$$
W^{H}-\delta W^{*}>0
$$

and

$$
W^{L}-\delta W^{*}>0 \text {. }
$$

Consequently, we obtain the same behavioral and welfare implications characterized in our earlier discussion. ${ }^{17}$

Our analysis shows how turn taking can mitigate conflict and coordination problems in the repeated Battle of the Sexes game even in the absence of communication and devices to correlate randomization. In this context, players randomize independently in the initial periods to resolve the questions of how they reach the turn taking path and who takes the good turn first. While we find that there is a welfare gain associated with turn taking, we also discover that the percentage of surplus attained is not close to the maximum of 100 , owing to the initial randomization, for some parameter combinations in Table 6. A natural direction for future research is to investigate, both qualitatively and quantitatively, how other complementary mechanisms such as cheap talk (see, for example, Farrell, 1987; Cooper et al., 1989; Farrell and Rabin, 1996) can further improve the welfare gain.

In this paper, we focus on the Battle of the Sexes game, since this game has been widely applied and since Luce and Raiffa (1957) refer to it in relation to turn taking under repeated interaction. Another direction for research is to understand the welfare gain of turn-taking behavior in other settings. For example, turn taking is observed in many situations in which common-pool

\footnotetext{
${ }^{16}$ Lau (2001) also uses this punishment strategy in a paper that provides a gametheoretic explanation for staggered contracts.

${ }^{17}$ Both this punishment strategy and the one discussed in Section 3 treat the two players symmetrically, irrespective of who cheats. Another strategy is to punish the player that deviates by restarting the turn-taking path at his bad turn with the other player starting at his good turn. This punishment strategy does not treat players symmetrically, but the punishment path is efficient. It can easily be shown that the no-deviation conditions for this punishment strategy are satisfied (for example, $\left.W^{L}-\delta W^{L}>0\right)$.
} 
resources, such as fishing spots in Turkey (Berkes, 1992), are assigned. Ostrom et al. (1994, pp. 58-61) suggest a "game of common-pool resource assignment" to capture, in the simplest fashion, a situation in which two fishermen independently decide to go to one of two fishing spots in their community. The good spot has a value of $h$, and the bad spot has a value of $l$, where $h>l>0$. If the two choose different spots, each will obtain the respective value of the spot. If they choose the same spot, they will split the value of the spot equally.

The assignment game described above, which also features interesting coordination and conflict problems, differs from the Battle of the Sexes game. For example, in one version of the one-shot assignment game where the good fishing spot is "sufficiently more attractive" than the bad spot (i.e., $h>2 l$ ), there is a unique pure-strategy equilibrium that involves both players going to the good spot. In Lau and Mui (2005), we find that the TTIR equilibrium of this version of the repeated assignment game can only be supported as a subgame-perfect equilibrium if players are patient enough, unlike the repeated Battle of the Sexes game in which the TTIR equilibrium can be supported as a subgame-perfect equilibrium for any discount factor. It will be interesting to study how turn taking can lead to conflict-mitigating and coordinationenhancing benefits in the game of common-pool resource assignment, as well as in other games.

\section{Appendix}

Proof of (15). From (9) and (10), it is straightforward to show that

$$
W^{H}+W^{L}=h+l=2 \pi .
$$

Another way to represent (14) is

$$
W^{*}=\left(p^{*}\right)^{2}\left(\delta W^{*}\right)+p^{*}\left(1-p^{*}\right) W^{H}+p^{*}\left(1-p^{*}\right) W^{L}+\left(1-p^{*}\right)^{2}\left(\delta W^{*}\right) .
$$

After simplification, we obtain

$$
W^{*}=\frac{p^{*}\left(1-p^{*}\right)\left(W^{H}+W^{L}\right)}{1-\delta\left[\left(p^{*}\right)^{2}+\left(1-p^{*}\right)^{2}\right]} .
$$

It is easy to see that the second equality of (14) leads to the first equality of (15). Substituting (9), (A1) and (A2) into the first equality of (15) leads to the second equality of (15). 
Proof of Proposition 1. Based on (15) and (A2), we define the function $f(p)$ for $0 \leq p \leq 1$ as

$$
f(p)=\frac{W^{H}-\delta W(p)}{\left[W^{H}-\delta W(p)\right]+\left[W^{L}-\delta W(p)\right]},
$$

where $W(p)$ is defined in (13). ${ }^{18}$ It is easy to see that $p^{*}$ in $(15)$ is a fixed point of function $f(p)$.

Using (9), (10) and (13), it can be shown that for $0 \leq p \leq 1$,

$$
W^{H}>W(p)>\delta W(p),
$$

and

$$
W^{L}=(1-\delta) l+\delta W^{H}>\delta W(p) .
$$

We know from (9) and (10) that $W^{H}>W^{L}$. Combining it with (A3), (A4) and (A5), we conclude that for all $0 \leq p \leq 1$,

$$
0.5<f(p)<1 .
$$

Thus, $f(p)$ is a continuous mapping from the compact set $[0,1]$ to itself. Applying the Brouwer's Fixed Point Theorem, we know that this function has a fixed point. That is, there exists a $p \in[0,1]$ such that $f(p)=p$. Moreover, $f(p)=p$ does not hold at $p=0$ or $p=1$, since $f(0) \neq 0$ and $f(1) \neq 1$ according to (A6). Combining the above results, we conclude that the solution to $(15)$ exists in the interval $(0,1)$. We denote the solution by $p^{*}$.

To show the uniqueness of $p^{*}$, we use (A3) and (13) to obtain

$$
f^{\prime}(p)=\frac{\delta\left(W^{H}-W^{L}\right)}{\left[W^{H}+W^{L}-2 \delta W(p)\right]^{2}} \frac{2(1-2 p)(1-\delta) \pi}{\left\{1-\delta\left[p^{2}+(1-p)^{2}\right]\right\}^{2}} .
$$

According to (A6), $f(p)>0.5$ for $p \in[0,0.5]$. Therefore, $f(p)$ will not intersect with the 45-degree line in this interval. On the other hand, $f(p)$ decreases when $p$ increases from 0.5 to 1 according to $(\mathrm{A} 7)$, and $0.5<f(0.5)<1$ and $0.5<f(1)<1$ according to (A6). Thus, $f(p)$ must intersect exactly once with the 45 -degree line when $p \in(0.5,1)$. We conclude that $p^{*} \in(0,1)$ is unique and it satisfies (16).

Simplifying (15) leads to

$$
\delta(\theta-1)\left(p^{*}\right)^{2}+(1+2 \delta+\theta) p^{*}-(\delta+\theta)=0 .
$$

\footnotetext{
${ }^{18}$ Note that we restrict $0<p<1$ in the main text, since the turn-taking phase will never be reached if both players choose $p=0$ or $p=1$ in the randomization phase. We extend the domain of $f(p)$ in (A3) to include $p=0$ and $p=1$ in order to apply well-known mathematical results.
} 
Applying the quadratic formula, it is straightforward (but tedious) to show that only one of the two roots to (A8) is between 0 and 1 , and it is given by (17).

Proof of Proposition 2. We know from (16) that $p^{*}>0.5$ and from (18) that $q^{*}<1$. To show $p^{*}<q^{*}$, we proceed in two steps. First, we show that

$$
\frac{h+l}{1+\delta}>W^{*}
$$

The proof is as follows. Because of $\delta<1$, (A1), (A2) and

$$
\left(p^{*}\right)^{2}+\left(1-p^{*}\right)^{2}+2 p^{*}\left(1-p^{*}\right)=1,
$$

we have

$$
\begin{aligned}
\frac{h+l}{1+\delta}-W^{*} & >\frac{h+l}{2}-W^{*}=\pi-\frac{2 p^{*}\left(1-p^{*}\right) \pi}{1-\delta\left[\left(p^{*}\right)^{2}+\left(1-p^{*}\right)^{2}\right]} \\
& =\frac{(1-\delta)\left[\left(p^{*}\right)^{2}+\left(1-p^{*}\right)^{2}\right] \pi}{1-\delta\left[\left(p^{*}\right)^{2}+\left(1-p^{*}\right)^{2}\right]}>0 .
\end{aligned}
$$

Second, we show that (A9) is equivalent to $p^{*}<q^{*}$. This is because, using (7), (9), (10) and (23), we have

$$
\begin{gathered}
p^{*}<q^{*} \\
\Longleftrightarrow \frac{p^{*}}{1-p^{*}}<\frac{q^{*}}{1-q^{*}} \\
\Longleftrightarrow \frac{W^{H}-\delta W^{*}}{W^{L}-\delta W^{*}}<\frac{h}{l} \\
\Longleftrightarrow h\left(W^{L}-\delta W^{*}\right)>l\left(W^{H}-\delta W^{*}\right) \\
\Longleftrightarrow h\left(\frac{l+\delta h}{1+\delta}\right)-l\left(\frac{h+\delta l}{1+\delta}\right)>(h-l) \delta W^{*} \\
\Longleftrightarrow \delta\left(h^{2}-l^{2}\right)>(h-l) \delta(1+\delta) W^{*} \\
\Longleftrightarrow \frac{h+l}{1+\delta}>W^{*} .
\end{gathered}
$$

Combining the above results, we prove Proposition 2.

Proof of Proposition 4. From (27) to (29), it can be shown that

$$
W_{m}^{*}=\frac{p_{m}^{*}\left(1-p_{m}^{*}\right)\left(W_{m}^{H}+W_{m}^{L}\right)}{1-\delta\left[\left(p_{m}^{*}\right)^{2}+\left(1-p_{m}^{*}\right)^{2}\right]}=\frac{2 p_{m}^{*}\left(1-p_{m}^{*}\right) \pi}{1-\delta\left[\left(p_{m}^{*}\right)^{2}+\left(1-p_{m}^{*}\right)^{2}\right]}
$$


Combining (A10) and the second equality of (29), we obtain

$$
p_{m}^{*}=\frac{W_{m}^{H}-\delta W_{m}^{*}}{\left(W_{m}^{H}-\delta W_{m}^{*}\right)+\left(W_{m}^{L}-\delta W_{m}^{*}\right)}=\frac{W_{m}^{H}-\delta\left\{\frac{2 p_{m}^{*}\left(1-p_{m}^{*}\right) \pi}{1-\delta\left[\left(p_{m}^{*}\right)^{2}+\left(1-p_{m}^{*}\right)^{2}\right]}\right\}}{W_{m}^{H}+W_{m}^{L}-2 \delta\left\{\frac{2 p_{m}^{*}\left(1-p_{m}^{*}\right) \pi}{1-\delta\left[\left(p_{m}^{*}\right)^{2}+\left(1-p_{m}^{*}\right)^{2}\right]}\right\}} .
$$

We define the function $g\left(p_{m}\right)$ for $0 \leq p_{m} \leq 1$ as

$$
g\left(p_{m}\right)=\frac{W_{m}^{H}-\delta W_{m}\left(p_{m}\right)}{\left[W_{m}^{H}-\delta W_{m}\left(p_{m}\right)\right]+\left[W_{m}^{L}-\delta W_{m}\left(p_{m}\right)\right]},
$$

where

$$
W_{m}\left(p_{m}\right)=\frac{2 p_{m}\left(1-p_{m}\right) \pi}{1-\delta\left[\left(p_{m}\right)^{2}+\left(1-p_{m}\right)^{2}\right]} .
$$

Note that $p_{m}^{*}$ in (A11) is a fixed point of function $g\left(p_{m}\right)$ in (A12).

It can be shown from (A13) that for $0 \leq p_{m} \leq 1$,

$$
W_{m}^{H}+W_{m}^{L}>2 \delta W_{m}\left(p_{m}\right) .
$$

We know from (27) and (28) that $W_{m}^{H}>W_{m}^{L}$. Combining it with (A12) and (A14), we know that for all $0 \leq p_{m} \leq 1,{ }^{19}$

$$
g\left(p_{m}\right)>0.5
$$

Next, we obtain

$$
g^{\prime}\left(p_{m}\right)=\frac{\delta\left(W_{m}^{H}-W_{m}^{L}\right)}{\left[W_{m}^{H}+W_{m}^{L}-2 \delta W_{m}\left(p_{m}\right)\right]^{2}} \frac{2\left(1-2 p_{m}\right)(1-\delta) \pi}{\left\{1-\delta\left[\left(p_{m}\right)^{2}+\left(1-p_{m}\right)^{2}\right]\right\}^{2}}
$$

According to (A15), $g\left(p_{m}\right)>0.5$ for $p_{m} \in[0,0.5]$. Therefore, $g\left(p_{m}\right)$ will not intersect with the 45-degree line in this interval. On the other hand, $g\left(p_{m}\right)$ decreases when $p_{m}$ increases from 0.5 to 1 according to (A16), $g(0.5)>0.5$ according to (A15), and $0.5<g(1)=\frac{W_{m}^{H}}{W_{m}^{H}+W_{m}^{L}}<1$. Thus, $g\left(p_{m}\right)$ must intersect exactly once with the 45 -degree line when $p_{m} \in(0.5,1)$. We conclude that $p_{m}^{*} \in(0,1)$ exists, is unique and it satisfies (30). This proves part (a).

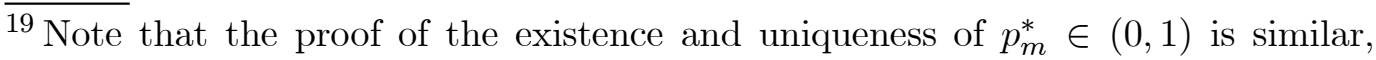
but not identical, to that in Proposition 1. This is because for multiple-period alternation, $g\left(p_{m}\right)$ may be greater than 1 , since $W_{m}^{L}$ may be smaller than $\delta W_{m}\left(p_{m}\right)$. 
To prove parts (b) and (c), we interpret $m$ as a continuous variable and use techniques in calculus. The second equality of (29) can be rewritten as

$p_{m}^{*}(m)\left[W_{m}^{L}(m)-\delta W_{m}^{*}\left(p_{m}^{*}(m)\right)\right]=\left[1-p_{m}^{*}(m)\right]\left[W_{m}^{H}(m)-\delta W_{m}^{*}\left(p_{m}^{*}(m)\right)\right]$,

where the dependence of $W_{m}^{H}$ on $m, W_{m}^{L}$ on $m, p_{m}^{*}$ on $m$, and $W_{m}^{*}$ on $p_{m}^{*}(m)$ are explicitly stated. It is clear from (A10) that $W_{m}^{*}$ depends on $m$ via $p_{m}^{*}$ only.

Differentiating the above expression with respect to $m$, and simplifying, give

$$
\frac{d p_{m}^{*}}{d m}=\frac{\frac{d W_{m}^{H}}{d m}-p_{m}^{*}\left[\frac{d\left(W_{m}^{H}+W_{m}^{L}\right)}{d m}\right]}{\left(W_{m}^{H}+W_{m}^{L}-2 \delta W_{m}^{*}\right)+\delta\left(1-2 p_{m}^{*}\right) \frac{d W_{m}^{*}}{d p_{m}^{*}}} .
$$

The sign of various components of (A17) are obtained as follows. We know from (27), (28), (30), (A13) and (A14) that

$$
\begin{gathered}
W_{m}^{H}+W_{m}^{L}-2 \delta W_{m}^{*}>0, \\
\frac{d W_{m}^{H}}{d m}=\frac{-(h-l) \delta^{m} \ln \delta}{\left(1+\delta^{m}\right)^{2}}>0 \\
\frac{d\left(W_{m}^{H}+W_{m}^{L}\right)}{d m}=\frac{d(2 \pi)}{d m}=0
\end{gathered}
$$

and

$$
\frac{d W_{m}^{*}}{d p_{m}^{*}}=\frac{2(1-\delta)\left(1-2 p_{m}^{*}\right) \pi}{\left\{1-\delta\left[\left(p_{m}^{*}\right)^{2}+\left(1-p_{m}^{*}\right)^{2}\right]\right\}^{2}}<0 .
$$

We conclude from (30), (A14a), (A18), (A19) and (A20) that

$$
\frac{d p_{m}^{*}}{d m}>0
$$

This proves part (b).

Since $W_{m}^{*}$ depends on $m$ via $p_{m}^{*}$ only, we obtain

$$
\frac{d W_{m}^{*}}{d m}=\left(\frac{d W_{m}^{*}}{d p_{m}^{*}}\right)\left(\frac{d p_{m}^{*}}{d m}\right)<0
$$

because of (A20) and (A21). This proves part (c). 


\section{References}

[1] Abreu, D. (1988), "On the Theory of Infinitely Repeated Games with Discounting," Econometrica, 56, 383-396.

[2] Berkes, F. (1992), "Success and Failure in Marine Coastal Fisheries of Turkey," in D. W. Bromley (ed.), Making the Commons Work: Theory, Practice, and Policy, 161-182. San Francisco: Institute for Contemporary Studies.

[3] Besen, S. and J. Farrell, (1994), "Choosing How to Compete: Strategies and Tactics in Standardization," Journal of Economic Perspectives, 8(2), 117-131.

[4] Bhaskar, V. (2000), "Egalitarianism and Efficiency in Repeated Symmetric Games," Games and Economic Behavior, 32, 247-262.

[5] Bornstein, G., D. Budescu and S. Zamir (1997), "Cooperation in Intergroup, N-person, and Two-Person Games of Chicken," Journal of Conflict Resolution, 41, 384-406.

[6] Cooper, R., D. V. DeJong, R. Forsythe and T. W. Ross (1989), "Communication in the Battle of the Sexes Game: Some Experimental Results," Rand Journal of Economics, 20, 568-587.

[7] Cooper, R., D. V. DeJong, R. Forsythe and T. W. Ross (1993), "Forward Induction in the Battle-of-the-Sexes Games," American Economic Review, 83(5), 1303-1316.

[8] Crawford, V. P. and H. Haller (1990), "Learning How to Cooperate: Optimal Play in Repeated Coordination Games," Econometrica, 58, 571-595.

[9] Dixit, A. and C. Shapiro (1986), "Entry Dynamics with Mixed Strategies," in L. G. Thomas (ed.), The Economics of Strategic Planning, 63-79. Lexington: Lexington Books.

[10] Dixit, A. and S. Skeath (2004), Games of Strategy, Second Edition. New York: W. W. Norton \& Company.

[11] Farrell, J. (1987), "Cheap Talk, Coordination, and Entry," Rand Journal of Economics, 18, 34-39.

[12] Farrell, J. and M. Rabin, (1996), "Cheap Talk," Journal of Economic Perspectives, 10, 103, 118.

[13] Friedman, J. W. (1994), "Introduction and Overview," in J. W. Friedman (ed.), Problems of Coordination in Economic Activity, 3-15. Boston: Kluwer Academic Publishers.

[14] Fudenberg, D. and E. Maskin (1986), "The Folk Theorem in Repeated Games with Discounting or with Incomplete Information," Econometrica, 54, 533-554.

[15] Fudenberg, D. and E. Maskin (1991), "On the Dispensability of Public Randomization in Discounted Repeated Games," Journal of Economic Theory, 53, 428-438. 
[16] Lau, S.-H. P. (2001), "Aggregate Pattern of Time-Dependent Adjustment Rules, II: Strategic Complementarity and Endogenous Nonsynchronization," Journal of Economic Theory, 98, 199-231.

[17] Lau, S.-H. P. and V.-L. Mui (2005), "Intratemporal Asymmetry, Intertemporal Efficiency and Turn Taking," Working Paper, University of Hong Kong and Monash University. (http://www.econ.hku.hk/ ${ }^{\sim}$ shlau/papers.html)

[18] Luce, R. D. and H. Raiffa (1957), Games and Decisions: Introduction and Critical Survey, New York: Wiley.

[19] Muller, R. A. and A. Sadanand (2003), "Order of Play, Forward Induction, and Presentation Effects in Two-Person Games," Experimental Economics, 6, 5-25.

[20] Ostrom, E., R. Gardner and J. Walker (with A. Agrawal, W. Blomquist, E. Schlager, and S. Y. Tang), (1994), Rules, Games, and Common-Pool Resources, Ann Arbor: University of Michigan Press.

[21] Sorin, S., (1986), "On Repeated Games with Complete Information," Mathematics of Operations Research, 11, 147-160. 
Table 1: A Battle of the Sexes Game

\begin{tabular}{|l|c|c|}
\hline \multicolumn{1}{|c|}{$\mathbf{1} \backslash \mathbf{2}$} & Ballet & Football \\
\hline Ballet & $(h, l)$ & $(0,0)$ \\
\hline Football & $(0,0)$ & $(l, h)$ \\
\hline
\end{tabular}

Table 2: Two Specifications of the Battle of the Sexes Game

(a) The $h$ and $l$ Specification

\begin{tabular}{|l|c|c|}
\hline $1 \backslash 2$ & Tough & Soft \\
\hline Tough & $(0,0)$ & $(h, l)$ \\
\hline Soft & $(l, h)$ & $(0,0)$ \\
\hline
\end{tabular}

Table 3: Payoff Matrix of the Repeated Battle of the Sexes Game

\begin{tabular}{|l|c|c|}
\hline \multicolumn{1}{|c|}{$1 \backslash 2$} & Playing Tough at Period 0 & Playing Soft at Period 0 \\
\hline Playing Tough at Period 0 & $\left(\delta W^{*}, \delta W^{*}\right)$ & $\left(W^{H}, W^{L}\right)$ \\
\hline Playing Soft at Period 0 & $\left(W^{L}, W^{H}\right)$ & $\left(\delta W^{*}, \delta W^{*}\right)$ \\
\hline
\end{tabular}

Table 4: Randomization Probability $\left(p^{*}\right)$ at the TTIR Equilibrium ${ }^{1}$

\begin{tabular}{|c|c|c|c|c|}
\hline$\delta \backslash \theta$ & 1.01 & 1.5 & 4 & 10 \\
\hline 0.5 & 0.5012 & 0.55 & 0.65 & 0.69 \\
\hline 0.78125 & 0.5009 & 0.534 & $\mathbf{0 . 6 0}$ & 0.63 \\
\hline 0.9 & 0.5007 & 0.529 & 0.58 & 0.61 \\
\hline 0.99 & 0.5006 & 0.525 & 0.57 & 0.60 \\
\hline \hline One-shot game & 0.5025 & $\mathbf{0 . 6 0}$ & $\mathbf{0 . 8 0}$ & 0.91 \\
\hline
\end{tabular}

1 The entries in the first four rows, which represent $p^{*}$ at the TTIR equilibrium of the repeated game, are calculated according to (17). For comparison, we also calculate $q^{*}$ at the mixed-strategy equilibrium of the one-shot game, which depends on $\theta$ only, according to (7). 
Table 5: Equilibrium Intertemporal Degree of Conflict $\left(\theta^{R}\right)^{1}$

\begin{tabular}{|c|c|c|c|c|}
\hline$\delta \backslash \theta$ & 1.01 & 1.5 & 4 & 10 \\
\hline 0.5 & 1.0050 & 1.22 & 1.82 & 2.27 \\
\hline 0.78125 & 1.0034 & 1.15 & $\mathbf{1 . 5 0}$ & 1.73 \\
\hline 0.9 & 1.0029 & 1.12 & 1.41 & 1.59 \\
\hline 0.99 & 1.0025 & 1.11 & 1.35 & 1.50 \\
\hline
\end{tabular}

1 The entries, which represent $\theta^{R}$ at the TTIR equilibrium of the repeated game, are calculated according to (23).

Table 6: Each Player's Payoff $\left(W^{*}\right)$ at the TTIR Equilibrium ${ }^{1}$

\begin{tabular}{|c|c|c|c|c|}
\hline$\delta \backslash \theta$ & 1.01 & 1.5 & 4 & 10 \\
\hline 0.5 & 66.67 & 66.22 & 62.78 & 59.60 \\
\hline 0.78125 & 82.05 & 81.91 & $\mathbf{8 0 . 8 4}$ & 79.85 \\
\hline 0.9 & 90.91 & 90.85 & 90.42 & 90.02 \\
\hline 0.99 & 99.01 & 99.00 & 98.97 & 98.93 \\
\hline \hline One-shot game & 49.999 & $\mathbf{4 8 . 0 0}$ & $\mathbf{3 2 . 0 0}$ & 16.53 \\
\hline
\end{tabular}

1 The entries in the first four rows, which represent $W^{*}$ at the TTIR equilibrium of the repeated game, are calculated according to (A2) in the Appendix. For comparison, we also calculate $U^{*}$ at the mixed-strategy equilibrium of the one-shot game according to (8). All entries are based on $\pi=100$.

Table 7: Conflict-Mitigating and Coordination-Enhancing Benefits of Turn Taking ${ }^{1}$

\begin{tabular}{|c|c|c|c|c|}
\hline$\delta \backslash \theta$ & 1.01 & 1.5 & 4 & 10 \\
\hline 0.5 & $0.0009(0 \%)$ & $1.50(8 \%)$ & $13.75(45 \%)$ & $25.92(60 \%)$ \\
& $16.67(100 \%)$ & $16.72(92 \%)$ & $17.03(55 \%)$ & $17.15(40 \%)$ \\
\hline 0.78125 & $0.0011(0 \%)$ & $1.77(5 \%)$ & $\mathbf{1 6}(\mathbf{3 3 \%})$ & $29.90(47 \%)$ \\
& $32.05(100 \%)$ & $32.15(95 \%)$ & $\mathbf{3 2 . 8 4 ( 6 7 \% )}$ & $33.42(53 \%)$ \\
\hline 0.9 & $0.0011(0 \%)$ & $1.83(4 \%)$ & $16.56(28 \%)$ & $30.90(42 \%)$ \\
& $40.91(100 \%)$ & $41.02(96 \%)$ & $41.86(72 \%)$ & $42.59(58 \%)$ \\
\hline 0.99 & $0.0012(0 \%)$ & $1.87(4 \%)$ & $16.89(25 \%)$ & $31.48(38 \%)$ \\
& $49.01(100 \%)$ & $49.13(96 \%)$ & $50.07(74 \%)$ & $50.92(62 \%)$ \\
\hline
\end{tabular}

${ }^{1}$ For each cell, the top and bottom terms represent, respectively, the conflict-mitigating and coordination-enhancing benefits. They are calculated according to (25) and (26) respectively. The figures inside the parentheses represent the proportion of a particular welfare gain. 


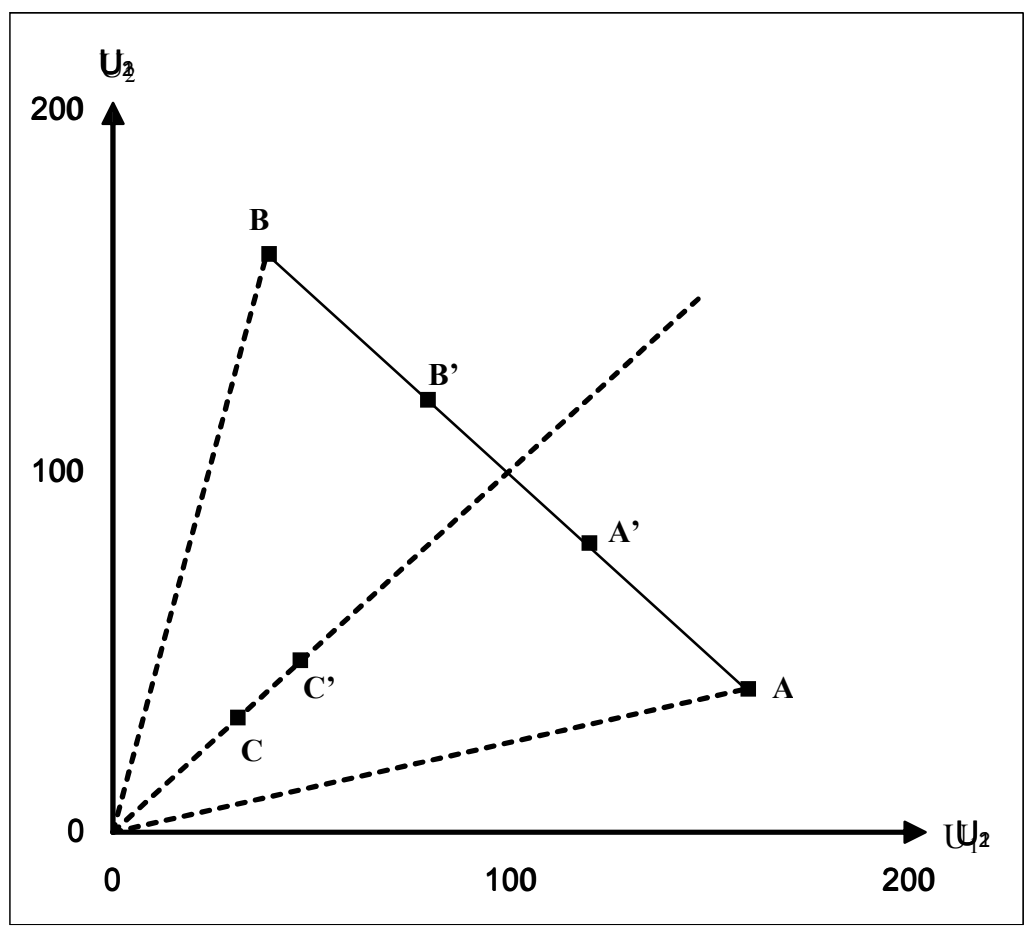

$A:\left(\frac{2 \theta \pi}{1+\theta}, \frac{2 \pi}{1+\theta}\right)$

$B:\left(\frac{2 \pi}{1+\theta}, \frac{2 \theta \pi}{1+\theta}\right)$

$C:\left(\frac{2 \theta \pi}{(1+\theta)^{2}}, \frac{2 \theta \pi}{(1+\theta)^{2}}\right)$

$A^{\prime}:\left(\frac{2 \theta^{\prime} \pi}{1+\theta^{\prime}}, \frac{2 \pi}{1+\theta^{\prime}}\right)$

$B^{\prime}:\left(\frac{2 \pi}{1+\theta^{\prime}}, \frac{2 \theta^{\prime} \pi}{1+\theta^{\prime}}\right)$

$C^{\prime}:\left(\frac{2 \theta^{\prime} \pi}{\left(1+\theta^{\prime}\right)^{2}}, \frac{2 \theta^{\prime} \pi}{\left(1+\theta^{\prime}\right)^{2}}\right)$

$\theta=4\left(q^{*}=0.8\right)$

$\theta^{\prime}=1.5\left(q^{*}=0.6\right)$

Figure 1: Two One-shot Battle of the Sexes Games with Different Degrees of Conflict

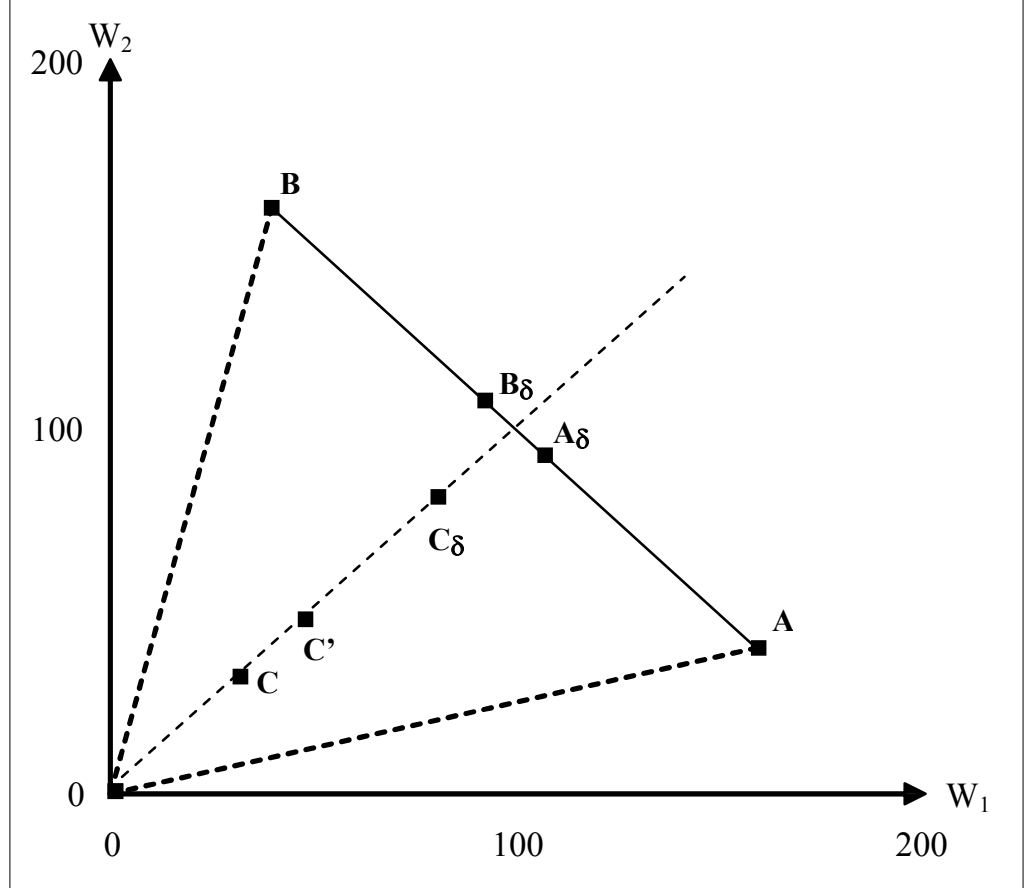

$$
\begin{aligned}
& A_{\delta}:\left(W^{H}, W^{L}\right) \\
& B_{\delta}:\left(W^{L}, W^{H}\right) \\
& C_{\delta}:\left(W^{*}, W^{*}\right) \\
& \delta=\frac{25}{32}=0.78125\left(p^{*}=0.6\right)
\end{aligned}
$$

Figure 2: Repeated Battle of the Sexes Game with the Same Degree of Conflict $(\theta=4)$ 